\title{
Research
}

\section{Effect of isoniazid prophylaxis on mortality and incidence of tuberculosis in children with HIV: randomised controlled trial}

\author{
Heather J Zar, Mark F Cotton, Stanzi Strauss, Janine Karpakis, Gregory Hussey, H Simon Schaaf, Helena Rabie, Carl J \\ Lombard
}

\begin{abstract}
Objectives To investigate the impact of isoniazid prophylaxis on mortality and incidence of tuberculosis in children with HIV. Design Two centre prospective double blind placebo controlled trial.

Participants Children aged $\geq 8$ weeks with HIV.

Interventions Isoniazid or placebo given with co-trimoxazole either daily or three times a week.

Setting Two tertiary healthcare centres in South Africa.

Main outcome measures Mortality, incidence of tuberculosis, and adverse events.

Results Data on 263 children (median age 24.7 months) were available when the data safety monitoring board recommended discontinuing the placebo arm; 132 (50\%) were taking isoniazid. Median follow-up was 5.7 (interquartile range 2.0-9.7) months. Mortality was lower in the isoniazid group than in the placebo group $(11(8 \%)$ v $21(16 \%)$, hazard ratio $0.46,95 \%$ confidence interval 0.22 to $0.95, \mathrm{P}=0.015$ ) by intention to treat analysis. The benefit applied across Centers for Disease Control clinical categories and in all ages. The reduction in mortality was similar in children on three times a week or daily isoniazid. The incidence of tuberculosis was lower in the isoniazid group (5 cases, $3.8 \%$ ) than in the placebo group (13 cases, 9.9\%) (hazard ratio $0.28,0.10$ to $0.78, \mathrm{P}=0.005$ ). All cases of tuberculosis confirmed by culture were in children in the placebo group. Conclusions Prophylaxis with isoniazid has an early survival benefit and reduces incidence of tuberculosis in children with HIV. Prophylaxis may offer an effective public health intervention to reduce mortality in such children in settings with a high prevalence of tuberculosis.

Trial registration. Clinical Trials NCT00330304
\end{abstract}

\section{Introduction}

Tuberculosis and HIV are dual pandemics in children in sub-Saharan Africa. Tuberculosis accelerates the course of HIV, increasing morbidity, mortality, and the frequency of opportunistic infections. ${ }^{1-4}$ It is an important cause of acute and chronic pneumonia in African children with $\mathrm{HIV}^{5-7}$ and is responsible for a major proportion of mortality. ${ }^{8}$ Infection with Mycobacterium tuberculosis confirmed by culture has been found in about $8 \%$ of children with HIV admitted to hospital for pneumonia in areas with high prevalence of tuberculosis and HIV. ${ }^{5-7}$ In a postmortem study of Zambian children dying from respiratory disease $M$ tuberculosis was found in 18\% of children with HIV; in children older than 12 months with HIV, tuberculosis was second to pyogenic pneumonia as a cause of death.
Prevention of tuberculosis in children with HIV through prophylaxis with isoniazid may be effective in reducing mortality in areas with a high prevalence of tuberculosis. In studies of adults with HIV, prophylaxis with isoniazid significantly reduced the incidence of tuberculosis and produced a favourable trend in mortality in those with a positive result on a tuberculin skin test. $^{9-11}$ The effect of such prophylaxis in children, however, is unknown.

We investigated the effect of isoniazid prophylaxis on mortality in children with HIV living in an area with high tuberculosis prevalence. We also looked at the incidence of tuberculosis, the susceptibility of $M$ tuberculosis isolates, the occurrence of toxicity, and the impact of two different prophylactic regimens.

\section{Methods}

We carried out a prospective double blind placebo controlled trial of isoniazid versus placebo given with co-trimoxazole (CTX) either daily or three times a week in children with HIV in two centres in Cape Town, South Africa. The study started in January 2003; the placebo arm of the study was ended on 17 May 2004 on the recommendation of the data safety monitoring board (DSMB) on the basis of the results of interim analyses.

\section{Participants}

Participants were children aged $\geq 8$ weeks with HIV who were attending the Red Cross Children's Hospital, University of Cape Town, or Tygerberg Children's Hospital, Stellenbosch University. Additional inclusion criteria were weight $\geq 2.5 \mathrm{~kg}$, access to transport, and informed consent from a parent or legal guardian. Exclusion criteria were chronic diarrhoea, current use or need for prophylaxis with isoniazid, previous hypersensitivity reaction to isoniazid or to sulphur drugs, severe anaemia (haemoglobin $<70 \mathrm{~g} / \mathrm{l}$ ), neutropenia (absolute neutrophil count $<400$ cells $/ \mu \mathrm{l}$ ), thrombocytopenia (platelet count $<50000 \times 10^{9} / \mathrm{l}$ ), or non-reversible renal failure. Children who were receiving highly active antiretroviral therapy (HAART) were eligible for enrolment if they had been stable on treatment for two to three months. Children were enrolled during working hours from Monday to Friday. Written informed consent was obtained from a parent or legal guardian.

Researchers took each child's history, carried out a physical examination, and collected sociodemographic, clinical, and laboratory data at enrolment. Children were seen by the study team every four weeks for the first six months then every six weeks for the next six months and then every two to three months, depending on the medical and social circumstances. 


\section{Assignment}

Pharmacists in each site labelled the trial drugs with sequential study numbers according to variable blocked randomisation lists prepared by the trial statistician. At enrolment children were also allocated a sequential study number by the research nurse and were then randomly assigned to one of the treatment combinations. Study investigators were blinded to the assignment. The research pharmacist dispensed the drugs.

\section{Prophylaxis}

Children were randomised to receive prophylaxis with co-trimoxazole either daily or three times a week on Monday, Wednesday, and Friday. As recommended by current South African guidelines, prophylaxis $(5 \mathrm{mg} / \mathrm{kg} /$ dose of the trimethoprim component) was given until the age of 12 months after which it was continued in those with clinical category B or C disease (according to the Centers for Disease Control), in those with severe immunological impairment (CD4 count of $<15 \%$ total lymphocyte count), or in those who had previous episode of Pneumocystis jirovecii pneumonia (PCP). The dose of isoniazid (100 mg tablets, Be-Tabs Pharmaceuticals, Johannesburg, South Africa) was $10 \mathrm{mg} / \mathrm{kg} /$ day with a variability of $8-12 \mathrm{mg} / \mathrm{kg}$ depending on whether half or quarter tablets were required. Placebo was manufactured to have an identical appearance to isoniazid tablets. Children received isoniazid or placebo according to the frequency of the co-trimoxazole schedule. The duration of prophylaxis was planned for two years but was subject to review depending on interim results.

\section{Other medication}

Multivitamin supplementation and immunisations were given according to a standard protocol. HAART was not widely available but was obtained for some children through participation in pharmaceutical trials or charitable donations. Standard guidelines for HAART in children in the Western Cape were developed during the study period; although these were not yet widely implemented, some children were able to access HAART according to medical and social criteria.

\section{Investigations}

HIV status was assessed at enrolment by two enzyme linked immunosorbent assays (Abbott AxSYM HIV antibody/antigen ELISA) in those aged $>15$ months and by polymerase chain reaction (Amplicor HIV-1, Roche Diagnostic Systems) in younger children. The CD4 cell count and percentage was measured at study entry and every six months. Full blood count, renal function (urea, creatinine), and alanine transaminase (ALT) were measured at baseline and every six months. A screening tuberculin skin test (PPD, 2 TU RT23, Staten Serum Institut, Copenhagen, Denmark) was done on enrolment and repeated every six months if previous results were negative. A positive result was regarded as $\geq 5 \mathrm{~mm}$ transverse induration. Children underwent screening chest radiography at enrolment and thereafter every six months. Blood tests, tuberculin skin tests, and chest radiography were performed more frequently if clinically indicated. In addition, for children receiving HAART, full blood count and alanine transaminase were measured one and three months after randomisation and thereafter every six months or more frequently if clinically indicated. If children were admitted to hospital or were ill between study visits, we took a detailed history and carried out clinical examination and laboratory tests as clinically indicated. The hospital or clinic records were obtained whenever possible for children who died; in the absence of these a verbal autopsy was performed when feasible.

\section{Diagnosis of tuberculosis}

Children were screened for tuberculosis on enrolment as described above. Any child who developed clinical signs of a lower respiratory tract infection underwent a tuberculin skin test and chest radiography. When chest radiography yielded abnormal results, three sequential gastric aspirates and sputum induction with nebulised hypertonic saline ${ }^{12}$ were performed for acid fast staining and $M$ tuberculosis culture. Additional specimens were sent for $M$ tuberculosis staining and culture as clinically indicated. Children were classified as having confirmed tuberculosis if they were culture positive for $M$ tuberculosis. Probable pulmonary tuberculosis was diagnosed when chest radiography suggested tuberculosis (lymphadenopathy, miliary pattern, pleural effusion, bronchial compression, or parenchymal infiltrate) and the child had at least one of: a positive tuberculin skin test result, a history of a close contact with tuberculosis, loss of weight or failure to gain weight within the previous three months, or a positive smear result for acid fast bacilli. The diagnosis of probable tuberculosis was subject to independent review by a blinded investigator.

Children with confirmed or probable tuberculosis were randomised at enrolment but isoniazid or placebo was started only after they had finished standard tuberculosis treatment. Children who developed confirmed or probable tuberculosis during the study were unblinded; those in the placebo group were given standard tuberculosis treatment while those in the isoniazid group were treated with four drugs (isoniazid, rifampicin, pyrazinamide, and ethambutol or ethionamide). Treatment was modified according to the antimicrobial susceptibility of cultured isolates.

\section{Toxicity}

Clinical or laboratory events were graded 1 to 4 according to the toxicity criteria of the National Institutes of Health's division of AIDS (DAIDS). A grade 3 or 4 reaction was considered an important adverse event and managed according to a standard protocol.

\section{Sample size and statistical analysis}

The primary outcome measure was mortality. Using a survival analysis approach, we estimated that a sample size in each group of 196 with a 0.050 level one sided log rank test for equality of survival curves would provide $80 \%$ power to detect the difference between an isoniazid-placebo mortality proportion at time $t$ of 0.100 and an isoniazid mortality proportion at time t of 0.050 (a constant hazard ratio of 0.769 ), assuming no dropouts before time t. Therefore, with an assumed 10\% dropout rate, the estimated required sample size was 216 children per arm or a total sample size of 432 .

All analyses were by intention to treat. We used the Kaplan-Meier method to analyse the time to event outcomes, made comparisons with a one sided log rank test, and used Cox proportional hazards regression to estimate hazard ratios after confirming the validity of the proportional hazards assumption. This assumption was tested with the Grambsch and Therneau test. $^{13}$ We did subgroups comparisons for severity of disease, dose, age, and study site to assess the consistency of the intervention effect. Tests for heterogeneity were done with the Cox regression model, except for tuberculin positivity as there were too few events in this subgroup. Anthropometric measurements were standardised with reference to standards from the National Center for Health Statistics. 


\section{Data safety monitoring board}

A safety monitoring board comprising international and South African experts reviewed and monitored the study at regular intervals. Interim data analyses were undertaken every three to six months to ensure the safety of the study and review progress.

\section{Results}

At the first meeting of the monitoring board, data up to 30 September 2003 were analysed. Of the 129 children enrolled 13/61 died in the placebo group and 4/68 died in the isoniazid group $(\mathrm{P}=0.009)$. The second meeting of the board in April 2004 considered data up to 30 December 2003. At that time, of the 148 children enrolled, 16 died in the placebo group and five in the isoniazid group $(\mathrm{P}=0.002$ by intention to treat and $\mathrm{P}<0.001$ for on treatment analysis). Both of these analyses met the O'Brien-Fleming rule for stopping a study, which requires $\mathrm{P}<0.01 .{ }^{14}$ As soon as the board recommended it, we terminated the placebo arm of the study. At this time, 277 children were enrolled. We excluded 14 children from the analysis (10 tested negative for HIV, four were lost to follow-up within a month after randomisation) and included 263 children (146 (56\%) boys) in the analysis (fig 1). Of these, 132 were assigned to isoniazid (three times a week in 68 and daily in 64). Median follow-up time was 5.7 months (interquartile range 2.0-9.7 months).

Table 1 provides the baseline characteristics of the children. About half were younger than 24 months. Most children (231, $88 \%$ ) were symptomatic, either Centers for Disease Control clinical category B or C. The median CD4 percentage was 20\%; the proportion of moderate or severely immunosuppressed children was similar in both groups. Overall, children were malnourished with the median weight for age $\mathrm{z}$ score equal to -1.6 (interquartile range $-2.5--0.4$ ) and the median weight for height $\mathrm{z}$ score equal to $-0.2(-1.1-0.9)$. Forty one (16\%) children had a history of tuberculosis, with a similar number in both groups. Tuberculin skin test results were positive in $22(9 \%)$; these children had previously received either prophylaxis or treatment for tuberculosis. At enrolment, 23 (9\%) were receiving HAART, while $58(22 \%)$ started HAART during the trial. The number of children who received HAART during the trial was similar both groups (41 in isoniazid group and 40 in placebo group).
Table 1 Comparison of children in isoniazid prophylaxis and placebo groups at randomisation. Figures are medians (interquartile range) for continuous variables or percentage (number) of children as shown

\begin{tabular}{lccc} 
& Isoniazid $(\mathrm{n}=132)$ & Placebo $(\mathrm{n}=131)$ & Total $(\mathrm{n}=263)$ \\
\hline Age (months): & & & \\
\hline Median & $29.6(11.0-55.3)$ & $22.1(8.9-45.4)$ & $24.7(9.4-51.6)$ \\
\hline$<12$ months & $26 \%(35)$ & $32 \%(42)$ & $29 \%(77)$ \\
\hline $12-24$ months & $19 \%(25)$ & $20 \%(26)$ & $19 \%(51)$ \\
\hline$>24$ months & $55 \%(72)$ & $48 \%(63)$ & $51 \%(135)$ \\
\hline Boys & $55 \%(72)$ & $57 \%(74)$ & $56 \%(146)$ \\
\hline Co-trimoxazole prophylaxis: & & & \\
\hline Daily & $49 \%(64)$ & $46 \%(60)$ & $47 \%(124)$ \\
\hline Three times a week & $52 \%(68)$ & $54 \%(71)$ & $53 \%(139)$ \\
\hline Weight for age (z score) & -1.55 & $-1.62(-2.61--0.44)$ & -1.56 \\
& $(-2.47--0.44)$ & & $(-2.49--0.43)$ \\
\hline Weight for height (z score) & -0.07 & $-0.19(-1.14-0.92)$ & -0.15 \\
& $(-1.07-0.88)$ & & $(-1.08-0.88)$ \\
\hline
\end{tabular}

Centers for Disease Control classification :

\begin{tabular}{lrrrr}
\hline $\mathrm{N}$ & $1 \%(1)$ & $2 \%(2)$ & $1 \%(3)$ \\
\hline $\mathrm{A}$ & $10 \%(13)$ & $12 \%(16)$ & $11 \%(29)$ \\
\hline $\mathrm{B}$ & $66 \%(87)$ & $66 \%(86$ & $66 \%(173)$ \\
\hline $\mathrm{C}$ & $24 \%(31)$ & $21 \%(27)$ & $22 \%(58)$ \\
\hline Tuberculosis: & & & & \\
\hline Prior TB treatment & $17 \%(23)$ & $14 \%(18)$ & $16 \%(41)$ \\
\hline $\begin{array}{l}\text { Positive tuberculin skin } \\
\text { test }\end{array}$ & $12 \%(15 / 128)$ & $5 \%(7 / 129)$ & $9 \%(22 / 257)$ \\
\hline
\end{tabular}

test

\begin{tabular}{|c|c|c|c|}
\hline \multicolumn{4}{|l|}{ Immune classification: } \\
\hline 1 & $30 \% \quad(40)$ & $23 \% \quad(30 / 129)$ & $27 \%(70 / 261)$ \\
\hline 2 & $40 \% \quad(53)$ & $41 \% \quad(53 / 129)$ & $41 \%(106 / 261)$ \\
\hline 3 & $30 \% \quad(39)$ & $36 \% \quad(46 / 129)$ & $33 \% \quad(85 / 261)$ \\
\hline \multicolumn{4}{|l|}{ Laboratory tests: } \\
\hline $\begin{array}{l}\text { Alanine transaminase } \\
(\mathrm{U} / \mathrm{l})\end{array}$ & $23(7-30)(n=113)$ & $22(6-31)(n=111)$ & $30(22-43)$ \\
\hline White cell count $\left(10^{9} / l\right)$ & $\begin{array}{c}9.5(7.0-13.4) \\
(n=120)\end{array}$ & $\begin{array}{c}10.7 \begin{array}{c}(7.7-13.8) \\
(n=122)\end{array} \\
\end{array}$ & $10.3(7.2-13.4)$ \\
\hline CD4 (\% lymphocytes) & $\begin{array}{c}21(14-29) \\
(n=114)\end{array}$ & $19(14-27)(n=118)$ & $20(14-28)$ \\
\hline $\begin{array}{l}\text { Receiving HAART at } \\
\text { enrolment }\end{array}$ & $10 \%(13)$ & $8 \%(10)$ & $9 \% \quad(23)$ \\
\hline
\end{tabular}

HAART=highly active antiretroviral therapy.

\section{Effect on mortality}

Mortality (32 deaths in 263 children, 12\%) was lower in the isoniazid group than in the placebo group (11/132 (8\%) v 21/131 $(16 \%)$, hazard ratio $0.46,95 \%$ confidence interval 0.22 to 0.95 ,

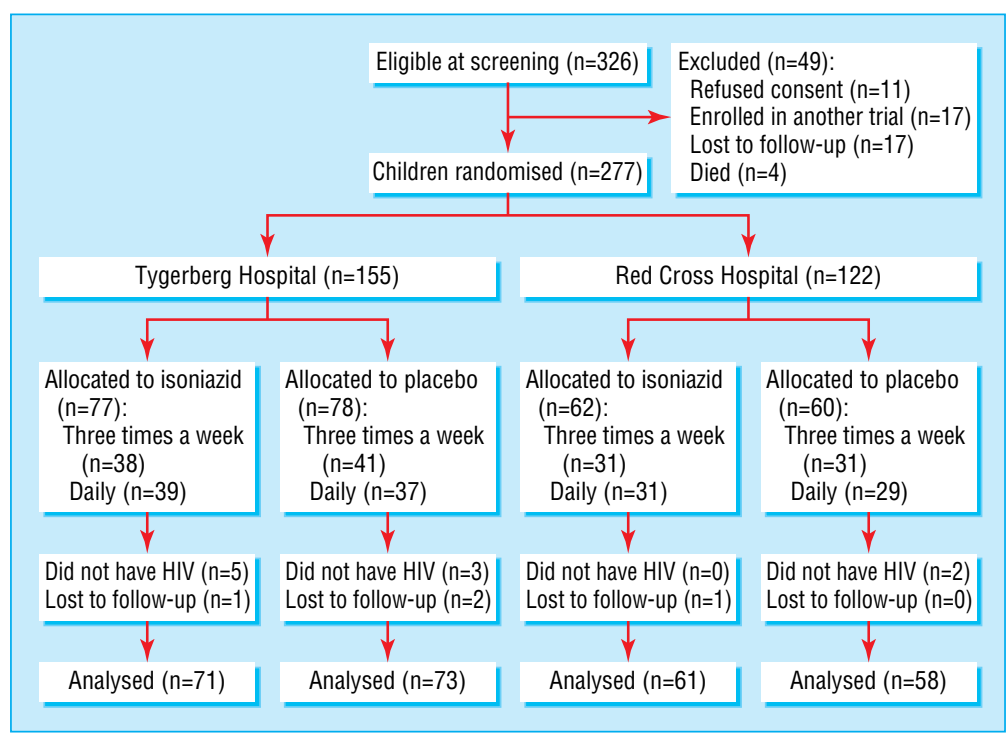

Fig 1 Allocation of participants and flow through trial 


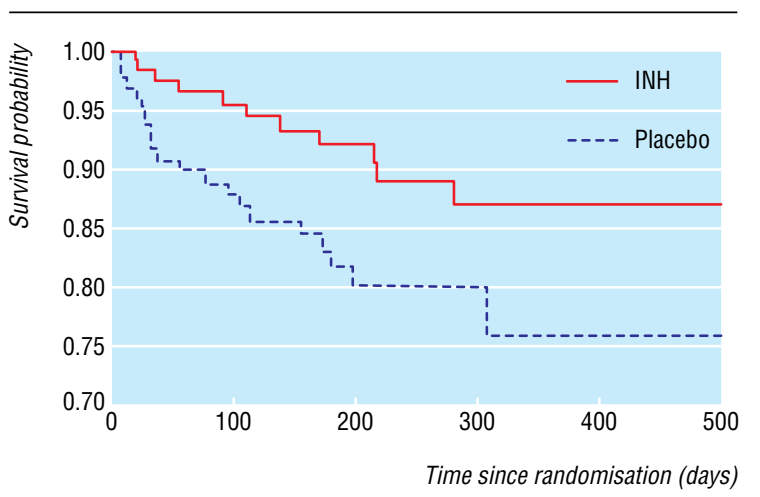

Fig 2 Survival in children on isoniazid (INH) or placebo

$\mathrm{P}=0.015$ for the one sided $\log$ rank test and $\mathrm{P}=0.226$ for the proportional hazards assumption; fig 2). The benefit applied to children across all categories of severity of clinical disease (test for heterogeneity $\mathrm{P}=0.933$ ) and in all ages (test for heterogeneity $\mathrm{P}=0.678$ ), table 2 . The reduction in mortality was similar in children assigned to isoniazid three times a week compared with every day (test of heterogeneity $\mathrm{P}=0.943$ ).

There were no deaths among children with positive results on tuberculin skin testing. The estimated hazard ratio for children negative for tuberculin was 0.51 (0.24 to 1.07). For children who received HAART at anytime during the study, three of 41 in the isoniazid group died with 195 months of exposure (the three children who died had a combined HAART exposure of 1.8 months) compared with none of the 40 children in the placebo group with 170 months of HAART exposure. The overall time without HAART was 577 months in the placebo group and 685 months in the isoniazid group. This converts to an

Table 2 Mortality and hazard ratios (HR) in children allocated to isoniazid prophylaxis or placebo

\begin{tabular}{|c|c|c|c|c|}
\hline & Isoniazid (\%) & Placebo (\%) & Total $(\%)$ & $\mathrm{HR}(95 \% \mathrm{Cl})$ \\
\hline Intention to treat & $11 / 132(8)$ & 21/131 (16) & $32 / 263$ (12) & $\begin{array}{c}0.46 \\
(0.22 \text { to } 0.95)\end{array}$ \\
\hline \multicolumn{5}{|l|}{ Frequency: } \\
\hline $\begin{array}{l}\text { Three times a } \\
\text { week }\end{array}$ & $5 / 68$ (7) & 9/71 (13) & 14/139 (10) & $\begin{array}{c}0.44 \\
(0.17 \text { to } 1.18)\end{array}$ \\
\hline Daily & $6 / 64(9)$ & $12 / 60$ (20) & 18/124 (15) & $\begin{array}{c}0.49 \\
(0.17 \text { to } 1.47)\end{array}$ \\
\hline \multicolumn{5}{|c|}{ Centers for Disease Control classification: } \\
\hline $\mathrm{A}+\mathrm{N}$ & $1 / 14(7)$ & $2 / 18(11)$ & $3 / 32$ (9) & $\begin{array}{c}0.82 \\
(0.07 \text { to } 9.22)\end{array}$ \\
\hline B & $6 / 87(7)$ & $11 / 86$ (13) & 17/173 (10) & $\begin{array}{c}0.45 \\
(0.16 \text { to } 1.21)\end{array}$ \\
\hline $\mathrm{C}$ & $4 / 31(13)$ & $8 / 27(30)$ & $12 / 58$ (21) & $\begin{array}{c}0.41 \\
(0.12 \text { to } 1.35)\end{array}$ \\
\hline \multicolumn{5}{|c|}{ Age group (months): } \\
\hline$<12$ & $7 / 35(20)$ & $13 / 42(31)$ & $20 / 77$ (26) & $\begin{array}{c}0.43 \\
(0.17 \text { to } 1.09)\end{array}$ \\
\hline $12-24$ & $3 / 25(12)$ & $5 / 26(19)$ & $8 / 51 \quad(16)$ & $\begin{array}{c}0.75 \\
\text { (0.18 to } 3.13)\end{array}$ \\
\hline$>24$ & $1 / 72(1)$ & $3 / 63(5)$ & $4 / 135$ (3) & $\begin{array}{c}0.26 \\
\text { (0.03 to } 2.49)\end{array}$ \\
\hline \multicolumn{5}{|c|}{ Tuberculin skin test result $(n=257)$ : } \\
\hline Positive & $0 / 15(0)$ & $0 / 7 \quad(0)$ & $0 / 22(0)$ & No estimate \\
\hline Negative & $11 / 113(10)$ & 20/122 (16) & $31 / 235$ (13) & $\begin{array}{c}0.51 \\
(0.24 \text { to } 1.07)\end{array}$ \\
\hline \multicolumn{5}{|c|}{ Receiving HAART at enrolment: } \\
\hline Yes & $0 / 13 \quad(0)$ & $0 / 10 \quad(0)$ & $0 / 23(0)$ & No estimate \\
\hline No & $11 / 119$ (9) & 21/121 (17) & $32 / 240$ (13) & $\begin{array}{c}0.46 \\
(0.22 \text { to } 0.95)\end{array}$ \\
\hline
\end{tabular}

HAART=highly active antiretroviral therapy.
Table 3 Incidence of tuberculosis in children allocated to isoniazid prophylaxis or placebo

\begin{tabular}{|c|c|c|c|c|}
\hline & $\begin{array}{c}\text { Isoniazid } \\
\mathrm{n}=132(\%)\end{array}$ & $\begin{array}{c}\text { Placebo } n=131 \\
(\%)\end{array}$ & $\begin{array}{c}\text { Total } n=263 \\
(\%)\end{array}$ & $\mathrm{HR}(95 \% \mathrm{Cl})$ \\
\hline Intention to treat & $5 / 132(4)$ & $13 / 131(10)$ & $18 / 263(7)$ & $0.28(0.10$ to 0.78$)$ \\
\hline \multicolumn{5}{|l|}{ Frequency of dose: } \\
\hline $\begin{array}{l}\text { Three times a } \\
\text { week }\end{array}$ & $3 / 68$ (4) & $5 / 71(7)$ & $8 / 139(6)$ & $0.45(0.11$ to 1.90$)$ \\
\hline Daily & $2 / 64(3)$ & $8 / 60(13)$ & $10 / 124(8)$ & $0.16(0.03$ to 0.76$)$ \\
\hline \multicolumn{5}{|c|}{ Centers for Disease Control classification: } \\
\hline $\mathrm{A}+\mathrm{N}$ & $0 / 14(0)$ & $1 / 18(6)$ & $1 / 32(3)$ & No estimate \\
\hline B & $4 / 87(5)$ & $11 / 86(13)$ & $15 / 173(9)$ & $0.22(0.07$ to 0.70$)$ \\
\hline C & $1 / 31 \quad(3)$ & $1 / 27(4)$ & $2 / 58(3)$ & $0.86(0.05$ to 13.8$)$ \\
\hline \multicolumn{5}{|c|}{ Age group (months): } \\
\hline$<12$ & $0 / 35(0)$ & $0 / 42(0)$ & $0 / 77(0)$ & No estimate \\
\hline $12-24$ & $2 / 25(8)$ & $5 / 26(19)$ & $7 / 51$ (14) & $0.50(0.10$ to 2.60$)$ \\
\hline$>24$ & $3 / 72(4)$ & $8 / 63(13)$ & $11 / 135(8)$ & $0.26(0.07$ to 0.98$)$ \\
\hline \multicolumn{5}{|c|}{ Tuberculin skin test result $(n=257)$ : } \\
\hline Positive & $0 / 15(0)$ & $1 / 7(14)$ & $1 / 22(5)$ & No estimate \\
\hline Negative & $5 / 113(4)$ & $12 / 122(10)$ & $17 / 235(7)$ & $0.32(0.11$ to 0.90$)$ \\
\hline \multicolumn{5}{|c|}{ Receiving HAART at enrolment: } \\
\hline Yes & $0 / 13(0)$ & $1 / 10(10)$ & $1 / 23(4)$ & No estimate \\
\hline No & $5 / 119$ (4) & $12 / 121(10)$ & $17 / 240(7)$ & $0.31(0.11$ to 0.87$)$ \\
\hline
\end{tabular}

HAART=highly active antiretroviral therapy.

annual non-HAART mortality of 43.6 per 100 in the placebo group and 14 per 100 in the isoniazid group.

In most children $(27,84 \%)$ the cause of death could be reliably determined. Clinical sepsis was the cause in 14 (44\%); 10 (31\%) had bacteraemia confirmed by culture. Most cultures were Gram negative bacteria (Klebsiella pneumoniae in four, acinetobacter in two, citrobacter in two, $K$ xanii in one, enterobacter species in one, Escherichia coli in one), and Staphylococcus aureus was isolated in two children. Three children had polymicrobial sepsis, and one child had concomitant cryptosporidial diarrhoea. Other causes of death included pneumonia $(7,22 \%)$, gastroenteritis (3, $9 \%$, and wasting syndrome, HIV encephalopathy with respiratory depression, and Burkitt's lymphoma in a single case each. In five $(16 \%)$ children the cause of death could not be ascertained: three died at home and two died at a local day health facility.

\section{Incidence of tuberculosis}

The incidence of confirmed or probable tuberculosis cases by intention to treat analysis (18 cases in 263 children, 7\%) was lower in the isoniazid group than in the placebo group $(5 / 132$ $(4 \%) v 13 / 131(10 \%)$, hazard ratio $0.28,0.10$ to $0.78, \mathrm{P}=0.005$ for the one sided $\log$ rank test and $\mathrm{P}=0.919$ for the proportional hazards assumption, table 3 ). The total child months of intent to treat follow-up in the two groups for the incidence of tuberculosis (total time from randomisation until a positive tuberculosis event or censoring event for each child) was 667 months in the placebo group and 839 months in the isoniazid group. This translated into 7.2 cases of tuberculosis annually per 100 children in the isoniazid group compared with 23.4 cases in the placebo group and an incidence rate ratio of 0.31 (0.09 to 0.91 ). The protective effect of isoniazid on incidence of tuberculosis occurred in all categories of severity of clinical disease in children aged $>1$ year and in both dose regimens (table 3 ). All five cases of tuberculosis confirmed by culture occurred in the placebo group. All $M$ tuberculosis isolates were sensitive to anti-tuberculosis drugs including isoniazid.

\section{Toxicity}

The incidence of grade 3 or 4 toxicity was low with five (4\%) in the isoniazid group and eight $(6.1 \%)$ in the placebo group. Of 
these, two were increases in alanine transaminase activity, both in the placebo group, while 11 were haematological events including neutropenia, thrombocytopenia, or anaemia. Alternative causes of haematological events included infections, other drugs, and HIV infection. No child required permanent discontinuation of trial drug. No cutaneous or neurological toxicity was observed. No grade 3 or 4 toxicity occurred among children receiving HAART.

\section{Discussion}

\section{Efficacy of isoniazid prophylaxis}

Isoniazid prophylaxis significantly reduced mortality in children with HIV who were living in an area with a high prevalence of tuberculosis. The impact on mortality was evident in all categories of clinical disease, across age groups, and for varying degrees of immune suppression. The effect on survival occurred within six months of the initiation of prophylaxis and was in addition to that provided by co-trimoxazole. Furthermore, isoniazid prophylaxis reduced the incidence of tuberculosis by about $70 \%$. The impact on survival and incidence of tuberculosis was similar for isoniazid three times a week or once a day. Few children were taking HAART at randomisation, reflecting the poor access and unaffordability of antiretroviral therapy for most children in sub-Saharan Africa, so we could not evaluate the impact of isoniazid prophylaxis on mortality in this subgroup. The three deaths that occurred in children taking isoniazid and HAART occurred soon after HAART initiation, suggesting that it was started too late in these children with advanced HIV disease.

The Western Cape area of South Africa has one of the highest incidences of tuberculosis in the world, with reported rates of $988 / 100000$ population in $2004^{15}$ and an estimated annual risk of infection of $3.8 \% .{ }^{16}$ Children aged $<15$ years contribute about $20 \%$ of the case load. ${ }^{17}$ In addition, the incidence of HIV infection, as reflected by the prevalence in pregnant women, has increased exponentially from $8.6 \%$ in 2001 to $15.4 \%$ in $2004 .{ }^{18} \mathrm{In}$ contrast with our findings, a Cochrane review of prophylaxis in adults with HIV did not find a significant reduction in mortality, although a favourable trend on survival was reported for adults with positive results on tuberculin skin test. ${ }^{11}$ Furthermore, our observed reduction in incidence of tuberculosis in children taking isoniazid prophylaxis was greater than that reported for adults with $\mathrm{HIV}^{11}$ and also occurred in children with negative tuberculin results. In contrast, chemoprophylaxis in adults with HIV has been found to be significantly effective only in those with positive results on tuberculin skin test, reducing the risk of active tuberculosis by about $60 \%{ }^{11}$

In our study, only a few children had positive results on tuberculin skin test. The impact of isoniazid on mortality and incidence of tuberculosis could therefore be reliably assessed only in children with a negative result on a tuberculin skin test, in whom we found a consistently protective effect of isoniazid. The high number of children with negative results on tuberculin skin tests may reflect anergy as a result of HIV mediated immunosuppression (most children had moderate or severe immunosuppression by CD4 counts), depressed cell mediated immunity because of malnutrition (most children were also malnourished), or early or lack of infection with $M$ tuberculosis. Tuberculosis in young children with HIV differs from adult disease in that it usually reflects primary infection and may often develop into severe or disseminated disease, which may cause death. $^{3}$ Although all children were screened carefully for tuberculosis, diagnosis is notoriously difficult in those with
$\mathrm{HIV}^{3}{ }^{12}$ raising the possibility that children with early or subclinical $M$ tuberculosis infection were not detected.

\section{Possible mechanisms of isoniazid efficacy}

The effect of isoniazid prophylaxis on incidence of tuberculosis may therefore have been because of treatment of early, subclinical, or latent $M$ tuberculosis infection. In addition, ongoing isoniazid treatment may have provided primary or secondary prophylaxis against infection. Recently it has been reported that treatment for latent tuberculosis with isoniazid (but not rifampicin) alters the immune response, resulting in an increase in the number of interferon $\gamma$ producing $\mathrm{T}$ cells within a month of therapy. ${ }^{19}$ Therefore isoniazid prophylaxis, by enhancing the host immune response, may provide longstanding protection. This mechanism may explain the long term protection (in excess of 19 years) provided by nine months of isoniazid prophylaxis in Alaskan people without HIV. ${ }^{19}{ }^{20}$ The ability of isoniazid to stimulate such immunity in people with HIV is not known. Alternatively, isoniazid may have effectively provided primary prophylaxis against $M$ tuberculosis in an area where children are continuously at high risk of infection. ${ }^{16}$

The mechanism whereby isoniazid prophylaxis improves survival in children with HIV is unclear but could occur in several ways. Co-infection with $M$ tuberculosis and HIV results in more rapid deterioration of immune dysfunction, viral replication, and progression of HIV. ${ }^{21}$ Such immune decline has been reported to result in more frequent, severe infections such as bacterial sepsis, ${ }^{22}$ which was the cause of death in most of our children. Tuberculosis, however, may also be a direct cause of mortality, as has been reported in a postmortem study of Zambian children in which tuberculosis was the second commonest cause of death in children aged $>1$ year with HIV who died from respiratory disease. ${ }^{8}$ As confirmation of $M$ tuberculosis by culture is difficult and positive in about a third of children with clinically suspected tuberculosis, and, as bacterial co-infection may occur, it is possible that tuberculosis was underdiagnosed in our study. ${ }^{12}$ A substantial number of children (22\%) died from clinical pneumonia, some of whom may have had tuberculosis. A limitation of our study is that we were unable to perform postmortem studies to investigate this. A further mechanism for the efficacy of isoniazid may be activity against organisms other than $M$ tuberculosis. For example, isoniazid in combination with rifampicin has been reported to be effective against leishmaniasis, ${ }^{23}$ and isoniazid has been shown to inhibit development of the malaria parasite. ${ }^{24}$ Current understanding of the molecular mechanism of isoniazid activity is incomplete, though the drug has been shown to interfere with fatty acid metabolism. ${ }^{25}$ Although isoniazid has not been shown to have activity against other bacteria, this pathway has been postulated to affect microbes such as $E$ coli and other gram negative bacteria. ${ }^{26}$ Furthermore, isoniazid blocks the lethality of endotoxin in mice $^{27}$ and is an efficient scavenger of free radicals. ${ }^{28}$ Finally, co-trimoxazole prophylaxis has been reported to improve survival in African children with $\mathrm{HIV}^{29}$; isoniazid may have an additive antimicrobial effect.

\section{Safety and tolerability}

The safety and tolerability of isoniazid prophylaxis was excellent, even in the subgroup of children who were taking HAART. Data from adults have confirmed the safety of isoniazid, with reported hepatotoxicity in only $0.3 \%$ of people treated for latent tuberculosis. ${ }^{30}$ The optimal duration of prophylaxis, however, is not known and long term studies are needed. Limited studies in adults with HIV suggest that prolonged use may be associated with longer protection. ${ }^{31}$ Careful screening of children for 
tuberculosis before prophylaxis is started is important. Reassuringly, the incidence of resistant $M$ tuberculosis infection did not increase in children on prophylaxis, suggesting that it did not promote the development of resistance. Ongoing monitoring of this is needed.

Most children with HIV currently live in sub-Saharan Africa, where tuberculosis is highly prevalent and where there is limited access to antiretroviral therapy. Mortality among these children is much higher than that in children with HIV in the developed world, with infant mortality and mortality in those aged $<5$ around $25 \%$ and $65 \%$, respectively. ${ }^{33}$ Prophylaxis with isoniazid offers an available, well tolerated, and effective means for improving survival in these children in addition to that provided by co-trimoxazole. Tuberculosis prophylaxis is cost effective, extends life expectancy, reduces the incidence of tuberculosis, and provides savings in medical and social costs in adults with HIV who are tuberculin skin test positive. ${ }^{34}$ Therefore, isoniazid prophylaxis may be an important public health intervention for children with HIV living in areas with high prevalence of tuberculosis, particularly when antiretroviral therapy is not available. Our results support the routine use of isoniazid prophylaxis in such children who cannot access HAART. Further studies in children of the cost efficacy of this intervention, the long term durability of protection, the efficacy in areas with low prevalence of tuberculosis, and the applicability of our findings to those receiving HAART is needed.

We thank the children and their caregivers for participating. The study team comprised P Apolles, N Dlaku, E Dobbels, T Fakir, C Ford, D Gray, M George, L Holt, T Jennings, A Joachim, B Leibbrandt, A Loggie, G Lottering, M Louw, I Mong, P Mtiya, D Nchuna, F Ngcokovana, V Nkondlala, K Orpen, H Smit, R Streicher, E Swanepoel, E Walters, and M A Wolff. The data and safety monitoring committee comprised J Kaplan (chair), W El Sadr, P Donald, and N Beyers.

Contributors: HJZ and MFC conceived the study, wrote the protocol and grant application, and supervised the study. SS and JK were trial physicians. HSS and GH assisted with design, supervision, and coordination of the study. HSS also reviewed clinical diagnoses. HR supervised trial management. CJL contributed to study design and was responsible for development of the database and statistical analysis. HZ drafted the paper and is guarantor, and all authors contributed to the final manuscript.

Funding: Rockefeller Foundation, USA.

Competing interests: None declared.

Ethical approval: Research and ethics committees of the Universities of Cape Town and Stellenbosch, South Africa.

\section{What is already known on this topic}

Prophylaxis with isoniazid significantly reduces the incidence of tuberculosis in adults with HIV and a positive tuberculin skin test result

There are no published data on the impact on mortality or incidence of tuberculosis in children with HIV

\section{What this study adds}

Prophylaxis with isoniazid in children significantly reduced mortality by about $50 \%$ and incidence of tuberculosis by about $70 \%$

The reduction in mortality occurred in all categories of clinical disease, in children in all age groups, and for varying degrees of immune suppression

Such prophylaxis may offer an effective public health intervention to reduce mortality in children with HIV
1 Palme IB, Gudetta B, Bruchfeld J, Muhe L, Giesecke J. Impact of human immunodeficiency virus 1 infection on clinical presentation, treatment outcome and survival in a cohort of Ethiopian children with tuberculosis. Pediatr Infect Dis J 2002;21:1053-61.

2 Mukadi YD, Wiktor SZ, Coulibaly IM, Coulibaly D, Mbengue A, Folquet AM, et al. Impact of HIV infection on the development, clinical presentation, and outcome of tuberculosis among children in Abidjan, Cote d'Ivoire. AIDS 1997;11:1151-8.

3 Chintu C, Mwaba P. Tuberculosis in children with human immunodeficiency virus infection. Int J Tuberc Lung Dis 2005;9:477-84.

4 Hesseling AC, Schaaf SH, Westra AE, Werschkull H, Donald PR, Beyers N, et al. Outcome of HIV-infected children with culture-confirmed tuberculosis. Arch Dis Child 2005;24:1171-4.

5 Jeena PM, Pillay P, Pillay T, Coovadia HM. Impact of HIV-1 co-infection on presentation and hospital-related mortality in children with culture proven pulmonary tuberculosis in Durban, South Africa. Int J Tuberc Lung Dis 2002;6:672-7.

6 Zar HJ, Hanslo D, Tannenbaum E, Klein M, Argent A, Eley B, et al. Aetiology and outcome of pneumonia in human immunodeficiency virus-infected children hospitalized in South Africa. Acta Paediatr 2001;90:119-25.

7 Madhi SA, Petersen K, Madhi A, Khoosal M, Klugman KP. Increased disease burden and antibiotic resistance of bacteria causing severe community-acquired lower respiratory tract infections in human immunodeficiency type 1-infected children. Clin Infect Dis 2000;31:170-6.

8 Chintu C, Mudenda V, Lucas S, Nunn A, Lishimpi K, Maswahu D, et al. Lung disease at necropsy in African children dying from respiratory illnesses: a descriptive necropsy study. Lancet 2002;360:985-90.

9 Wilkinson D, Squire SB, Garner P. Effect of preventive treatment for tuberculosis in adults infected with HIV: systematic review of randomised placebo controlled trials. BMJ 1998;317:625-9.

10 Bucher HC, Griffith LE, Guyatt GH, Sundre P, Naef M, Sendi P, et al. Isoniazid prophylaxis for tuberculosis in HIV infection: a meta-analysis of randomized controlled trials. AIDS 1999;13:501-7.

11 Woldehanna S, Volmink J. Treatment of latent tuberculosis infection in HIV infected persons. Cochrane Database Syst Rev 2006;(3):CD000171.

12 Zar HJ, Hanslo D, Apolles P, Swingler G, Hussey G. Comparison of induced sputum with gastric lavage for microbiologic confirmation of pulmonary tuberculosis in infants and young children-a prospective study. Lancet 2005;365:130-4.

13 Grambsch PM, Therneau TM. Proportional hazards test and diagnostics based on weighted residuals. Biometrika 1994;81:515-26.

14 Pocock SJ. When to stop a clinical trial. BMJ 1992;305:235-40.

15 Health Systems Trust Health Statistics. www.hst.org.za/healthstats/16/data (accessed 19 Oct 2006).

16 Beyers N, Michaelis I, Gie RP, Schaaf HS, Richardson M, Warren R. Transmission of tuberculosis to children in a high incidence area. Int J Tuberc Lung Dis 2001;5(suppl 1):S185

17 Soeters M, de Vries AM, Kimpen JLL, Donald PR, Schaaf HS. Clinical features and outcome in children admitted to a TB hospital in the Western Cape-the influence of HIV and drug resistance. $S$ Afr Med J 2005;95:602-6.

18 National Department of Health. National HIV and syphilis seroprevalence survey in South Africa 2004. Pretoria: National Department of Health, 2005.

19 Wilkinson KA, Kon OM, Newton SM, Meintjes G, Davidson RN, Pasvol G, et al. Effect of treatment of latent tuberculosis infection on the T cell response to Mycobacterium tuberculosis antigens. J Infect Dis 2006;193:354-9.

20 Comstock GW, Baum C, Snider DE. Isoniazid prophylaxis among Alaskan Eskimos: a final report of the bethel isoniazid studies. Am Rev Respir Dis 1979;119:827-30.

21 Toossi Z. Virological and immunological impact of tuberculosis on human immunodeficiency virus type I disease. J Infect Dis 2003;188:1146-55.

22 Whalen C, Horsburgh CR, Hom D, Lahart C, Simberkoff M, Ellner J. Accelerated course of human immunodeficiency virus infection after tuberculosis. Am J Resp Crit Care Med 1995;151:129-35.

23 Peters W, Lainson R, Shaw JJ, Robinson BL, Leao AF. Potentiating action of rifampicin and isoniazid against Leishmania mexicana amazonensis. Lancet 1981;i:1122-4.

24 Arai M, Alavi YI, Mendoza J, Billker O, Sinden RE. Isonicotinic acid hydrazide: an antituberculosis drug inhibits malarial transmission in the mosquito gut. Exp Parasitol 2004;106:30-6.

25 Ducasse-Cabanot S, Cohen-Gonsaud M, Marrakchi H, Nguyen M, Zerbib D, Bernadou $\mathrm{J}$, et al. In vitro inhibition of the Mycobacterium tuberculosis beta-ketoacyl-acyl carrier protein reductase MabA by isoniazid. Antimicrob Agents Chemother 2004;48:242-9.

26 Baldock C, de Boer GJ, Rafferty JB, Stuitje AR, Rice DW. Mechanism of action of diazaborines. Biochem Pharmacol 1998;55:1541-9.

27 Urbaschek R, Männel DN, Urbanczik R. Isoniazid protects mice against endotoxin lethality without influencing tumor necrosis factor synthesis and release. Antimicrob Agents Chemother 1991;35:1666-8.

28 Choi D, Leininger-Muller B, Kim YC, Leroy P, Siest G, Wellman M. Differential role of CYP2E1 binders and isoniazid on CYP2E1 protein modification in NADPHdependent microsomal oxidative reactions: free radical scavenging ability of isoniazid. Free Radic Res 2002;36:893-903.

29 Chintu C, Bhat GJ, Walker AS, Mulenga V, Sinyinza F, Lishimpi K, et al. Co-trimoxazole as prophylaxis against opportunistic infections in HIV-infected Zambian children (CHAP): a double-blind randomised placebo-controlled trial. Lancet 2004:364:186571.

30 LoBue PA, Moser KS. Use of isonaizid for latent tuberculosis infection in a public health clinic. Am J Resp Crit Care Med 2003;168:443-7.

31 Casado JL, Moreno S, Fortun A, Antela A, Quereda C, Navas E, et al. Risk factors for development of tuberculosis after isoniazid chemoprophylaxis in human immunodeficiency virus-infected patients. Clin Infect Dis J 2002:34:386-9.

32 Fitzgerald DW, Desvarieux M, Severe P, Joseph P, Johnson WD Jr, Pape JW. Effect of post-treatment isoniazid on prevention of recurrent tuberculosis in HIV-1-infected individuals: a randomised trial. Lancet 2000;356:1470-4.

33 Spira R, Lepage P, Msellati P, van de Perra P, Leroy V, Simonon A, et al. Natural history of human immunodeficiency virus type 1 infection in children: a five-year prospective study in Rwanda. Pediatrics 1999;104:e56.

34 Bell JC, Rose DN, Sacks HS. Tuberculosis preventive therapy for HIV-infected people in sub-Saharan Africa is cost-effective. AIDS 1999;13:1549-56.

(Accepted 29 September 2006) 
doi $10.1136 /$ bmj. 39000.486400 .55

School of Child and Adolescent Health, Red Cross Children's Hospital, University of Cape Town, South Africa

Heather J Zar associate professor

Stanzi Strauss research medical officer

Gregory Hussey professor

Department of Paediatrics and Child Health, Tygerberg Children's Hospital, Stellenbosch University, South Africa
Mark F Cotton associate professor

Janine Karpakis research medical officer

H Simon Schaaf associate professor

Helena Rabie clinical researcher

Biostatistics Unit, Medical Research Council, South Africa

Carl J Lombard senior statistician

Correspondence to: H Zar hzar@ich.uct.ac.za 